\title{
Nanotechnology approaches to crossing the blood-brain barrier and drug delivery to the CNS
} Gabriel A Silva

Address: Departments of Bioengineering and Ophthalmology, and Neurosciences Program, University of California, San Diego, 9415 Campus Point Drive, La Jolla, California 92037-0946, USA

Email: Gabriel A Silva - gsilva@ucsd.edu

from 2008 Drug Discovery for Neurodegeneration Conference

New York, USA. 5-6 February 2007. Washington, DC, USA. 4-5 February 2008

Published: 10 December 2008

BMC Neuroscience 2008, 9(Suppl 3):S4 doi:10.1 186/147I-2202-9-S3-S4

This article is available from: http://www.biomedcentral.com/I47I-2202/9/S3/S4

(C) 2008 Silva; licensee BioMed Central Ltd.

This is an open access article distributed under the terms of the Creative Commons Attribution License (http://creativecommons.org/licenses/by/2.0), which permits unrestricted use, distribution, and reproduction in any medium, provided the original work is properly cited.

\begin{abstract}
Nanotechnologies are materials and devices that have a functional organization in at least one dimension on the nanometer (one billionth of a meter) scale, ranging from a few to about 100 nanometers. Nanoengineered materials and devices aimed at biologic applications and medicine in general, and neuroscience in particular, are designed fundamentally to interface and interact with cells and their tissues at the molecular level. One particularly important area of nanotechnology application to the central nervous system (CNS) is the development of technologies and approaches for delivering drugs and other small molecules such as genes, oligonucleotides, and contrast agents across the blood brain barrier (BBB). The BBB protects and isolates CNS structures (i.e. the brain and spinal cord) from the rest of the body, and creates a unique biochemical and immunological environment. Clinically, there are a number of scenarios where drugs or other small molecules need to gain access to the CNS following systemic administration, which necessitates being able to cross the BBB. Nanotechnologies can potentially be designed to carry out multiple specific functions at once or in a predefined sequence, an important requirement for the clinically successful delivery and use of drugs and other molecules to the CNS, and as such have a unique advantage over other complimentary technologies and methods. This brief review introduces emerging work in this area and summarizes a number of example applications to CNS cancers, gene therapy, and analgesia.
\end{abstract}

\section{Introduction}

Nanotechnologies are materials and devices that have a functional organization in at least one dimension on the nanometer (one billionth of a meter) scale, ranging from a few to about 100 nanometers. Nanoengineered materials and devices aimed at biologic applications and medicine in general, and neuroscience in particular, are designed fundamentally to interface and interact with cells and their tissues at the molecular level. The potential of nanotechnological applications to biology and medicine arise from the fact that they exhibit bulk mesoscale and macroscale chemical and/or physical properties that are unique to the engineered material or device and not necessarily possessed by the molecules alone. This supports the development of nanotechnologies that can potentially carry out multiple specific functions at once or 
in a predefined sequence, which is an important property for the clinically successful delivery of drugs and other molecules to the central nervous system (CNS).

An ability to cross the blood-brain barrier (BBB) to deliver drugs or other molecules (for example, oligonucleotides, genes, or contrast agents) while potentially targeting a specific group of cells (for instance, a tumor) requires a number of things to happen together. Ideally, a nanodelivery-drug complex would be administered systemically (for example, intravenously) but would find the CNS while producing minimal systemic effects, be able to cross the BBB and correctly target cells in the CNS, and then carry out its primary active function, such as releasing a drug. These technically demanding obstacles and challenges will require multidisciplinary solutions between different fields, including engineering, chemistry, cell biology, physiology, pharmacology, and medicine. Successfully doing so will greatly benefit the patient. Although this ideal scenario has not yet been realized, a considerable body of work has been done to develop nanotechnological delivery strategies for crossing the BBB.

\section{Applications to drugs and other molecules}

A significant amount of work using nanotechnological approaches to crossing the BBB has focused on the delivery of antineoplastic drugs to CNS tumors. For example, radiolabeled polyethylene glycol (PEG)-coated hexadecylcyanoarcylate nanospheres have been tested for their ability to target and accumulate in a rat model of gliosarcoma [1]. Another group has encapsulated the antineoplasitc drug paclitaxel in polylactic co-glycolic acid nanoparticles, with impressive results. In vitro experiments with 29 different cancer cell lines (including both neural and nonneural cell lines) demonstrated targeted cytotoxicity 13 times greater than with drug alone [2]. Using a variety of physical and chemical characterization methods, including different forms of spectroscopy and atomic force microscopy, the investigators showed that the drug was taken up by the nanoparticles with very high encapsulation efficiencies and that the release kinetics could be carefully controlled. Research focusing on the delivery of many of the commonly used antineoplastic drugs is important because most of these drugs have poor solubility under physiologic conditions and require less than optimal vehicles, which can produce significant side effects.

In another example, various compounds - including neuropeptides such as enkephalins, the $N$-methyl-D-aspartate receptor antagonist MRZ 2/576, and the chemotherapeutic drug doxorubicin - have been attached to the surface of poly(butylcyanoacrylate) nanoparticles coated with polysorbate 80 [3-7]. The polysorbate on the surface of the nanoparticles adsorb apolipoproteins $\mathrm{B}$ and $\mathrm{E}$ and are taken up by brain capillary endothelial cells via receptormediated endocytosis. Nanoparticle-mediated delivery of doxorubicin is being explored in a rodent model of glioblastoma $[3,8]$. Importantly, recent work in a rat glioblastoma model revealed significant remission with minimal toxicity, setting the stage for potential clinical trials [8].

The delivery of other drugs is also being investigated. Dalargin is a hexapeptide analog of leucine-enkephalin containing D-alanine, which produces CNS analgesia when it is delivered intracerebroventricularly, but it has no analgesic effects if it is administered systemically, specifically because it cannot cross the BBB on its own $[9,10]$. [3H]Dalargin was conjugated to the same poly(butylcyanoacrylate) nanoparticles described above, injected systemically into mice, and demonstrated by radiolabeling to cross the BBB and accumulate in brain [10]. Other, similar studies have also demonstrated delivery of dalargin using polysorbate 80-coated nanoparticles [11]. Other polysorbate 80 nanoparticles have been chemically conjugated to the hydrophilic drug diminazenediaceturate (diminazene) and proposed as a novel treatment approach for second stage African trypanosomiasis [12]. In other work, PEG-treated polyalkylcyanoacrylate nanoparticles were shown to cross the BBB and accumulate at high densities in the brain in experimental autoimmune encephalomyelitis [13], a model of multiple sclerosis [14,15].

For other applications, molecules other than drugs must cross the $\mathrm{BBB}$ for therapeutic or diagnostic reasons, including oligonucleotides, genes, and contrast agents. Solid lipid nanoparticles consisting of microemulsions of solidified oil nanodroplets loaded with iron oxide and injected systemically into rats have been shown to cross the $\mathrm{BBB}$ and accumulate in the brain with long-lasting kinetics [16]. Iron oxides are classic superparamagnetic magnetic resonance imaging (MRI) contrast agents. Because iron oxides are insoluble in water, they must be delivered as modified colloids for clinical applications, which is usually achieved by coating them with hydrophilic molecules, such as dextrans [17]. Therefore, the delivery vehicle used is critical in determining the functional properties of the contrast agent. By taking advantage of the ability of these solid lipid nanoparticles to cross the $\mathrm{BBB}$, nanoparticles complexed with iron oxides may provide new ways to image the CNS using MRI.

Other work has focused on the delivery of oligonucleotides in an in vivo mouse model and an in vitro endothelial cell model, with the aim being to develop novel treatments for neurodegenerative disorders [18]. The investigators synthesized a nanogel consisting of crosslinked PEG and polyethylenimine that spontaneously encapsulated negatively charged oligonucleotides. In their 
in vivo model, they demonstrated that intravenous injections resulted in a 15 -fold accumulation of oligonucleotides in the brain after 1 hour, with a concurrent twofold decrease in accumulation in liver and spleen when compared with freely administered oligonucleotides (not encapsulated in nanogel particles).

A related area is the delivery of genes to the CNS for gene therapy. A tyrosine hydroxylase (TH) expression plasmid was delivered to the striatum of adult rats using PEG immunoliposome nanoparticles in order to normalize $\mathrm{TH}$ expression levels in the 6-hydroxydopamine rat model of Parkinson's disease [19]. Using specific antibodies to transferrin receptors conjugated to the nanoparticles, TH plasmids were shown to be expressed throughout the striatum.

\section{Conclusion}

Nanotechnology-based approaches to targeted delivery of drugs and other compounds across the BBB may potentially be engineered to carry out specific functions as needed. The drug itself - in other words the biologically active component being delivered, whatever that may be - constitutes one element of a nanoengineered complex. The rest of the complex is designed to carry out other key functions, including shielding the active drug from producing systemic side effects, being prematurely cleared or metabolized, crossing the $\mathrm{BBB}$, and targeting specific cells after it has gained access to the CNS. Implicitly, all of this must be achieved by any drug intended to have CNS effects, regardless of whether it is part of a nanoengineered complex.

An important advantage of a nanotechnological approach, as compared with the administration of free drug or the drug associated with a nonfunctional vehicle, is that these critical requirements do not need to be carried out by the active compound, but by supporting parts of the engineered complex. This allows the design of the active drug to be tailored for maximal efficacy. Currently, most nanoengineered systems for crossing the BBB take advantage of drugs that are already in clinical use and therefore have greater potential for reaching the clinic relatively quickly.

In addition to the delivery of drugs and other compounds across the $\mathrm{BBB}$ for therapeutic purposes, the ability to cross the BBB selectively and efficiently in animal models using nanoengineered technologies will have a significant impact on research that focuses on the normal physiology of the CNS and its pathology, by allowing targeted in vivo studies of specific cells and processes using methods that take advantage of the intact live organism. Ideally, methods for crossing the BBB will complement other nanote- chnological tools being developed to study the CNS, including quantum dot labeling and imaging [20].

\section{List of abbreviations used}

BBB: blood-brain barrier; CNS: central nervous system; PEG: polyethylene glycol.

\section{Competing interests}

The author declares that they have no competing interests.

\section{Acknowledgements}

Parts of this paper were adapted from a more detailed review on nanotechnology approaches for crossing the BBB written by the author [2I]. This work was supported by funds from NIH grant NINDS NS054736.

This article has been published as part of BMC Neuroscience Volume 9 Supplement 3, 2008: Proceedings of the 2007 and 2008 Drug Discovery for Neurodegeneration Conference. The full contents of the supplement are available online at http://www.biomedcentral.com/I47|-2202/9?issue=S3.

\section{References}

I. Brigger I, Morizet J, Aubert G, Chacun H, Terrier-Lacombe MJ, Couvreur P, Vassal G: Poly(ethylene glycol)-coated hexadecylcyanoacrylate nanospheres display a combined effect for brain tumor targeting. J Pharmacol Exp Ther 2002, 303:928-936.

2. Feng SS, Mu L, Win KY, Huang G: Nanoparticles of biodegradable polymers for clinical administration of paclitaxel. Curr Med Chem 2004, I I:413-424.

3. Gulyaev AE, Gelperina SE, Skidan IN, Antropov AS, Kivman GY, Kreuter J: Significant transport of doxorubicin into the brain with polysorbate 80-coated nanoparticles. Pharm Res 1999, 16:1564-1569.

4. Alyautdin RN, Petrov VE, Langer K, Berthold A, Kharkevich DA, Kreuter J: Delivery of loperamide across the blood-brain barrier with polysorbate 80-coated polybutylcyanoacrylate nanoparticles. Pharm Res 1997, 14:325-328.

5. Kreuter J, Alyautdin RN, Kharkevich DA, Ivanov AA: Passage of peptides through the blood-brain barrier with colloidal polymer particles (nanoparticles). Brain Res 1995, 674:17|-174.

6. Alyautdin RN, Tezikov EB, Ramge P, Kharkevich DA, Begley DJ, Kreuter J: Significant entry of tubocurarine into the brain of rats by adsorption to polysorbate 80 -coated polybutylcyanoacrylate nanoparticles: an in situ brain perfusion study. J Microencapsul 1998, 15:67-74.

7. Friese A, Seiller E, Quack G, Lorenz B, Kreuter J: Increase of the duration of the anticonvulsive activity of a novel NMDA receptor antagonist using poly(butylcyanoacrylate) nanoparticles as a parenteral controlled release system. Eur J Pharm Biopharm 2000, 49:103-109.

8. Steiniger SC, Kreuter J, Khalansky AS, Skidan IN, Bobruskin AI, Smirnova ZS, Severin SE, Uhl R, Kock M, Geiger KD, Gelperina SE: Chemotherapy of glioblastoma in rats using doxorubicinloaded nanoparticles. Int J Cancer 2004, 109:759-767.

9. Rousselle C, Clair P, Smirnova M, Kolesnikov Y, Pasternak GW, GacBreton S, Rees AR, Scherrmann JM, Temsamani J: Improved brain uptake and pharmacological activity of dalargin using a peptide-vector-mediated strategy. J Pharmacol Exp Ther 2003, 306:37I-376.

10. Alyaudtin RN, Reichel A, Lobenberg R, Ramge P, Kreuter J, Begley DJ: Interaction of poly(butylcyanoacrylate) nanoparticles with the blood-brain barrier in vivo and in vitro. J Drug Target 200I, 9:209-221.

II. Schroeder U, Sommerfeld P, Ulrich S, Sabel BA: Nanoparticle technology for delivery of drugs across the blood-brain barrier. $J$ Pharm Sci 1998, 87: I 305-1307.

12. Olbrich C, Gessner A, Kayser O, Muller RH: Lipid-drug-conjugate (LDC) nanoparticles as novel carrier system for the hydrophilic antitrypanosomal drug diminazenediaceturate. J Drug Target 2002, 10:387-396. 
13. Calvo P, Gouritin B, Villarroya H, Eclancher F, Giannavola C, Klein C, Andreux JP, Couvreur P: Quantification and localization of PEGylated polycyanoacrylate nanoparticles in brain and spinal cord during experimental allergic encephalomyelitis in the rat. Eur J Neurosci 2002, 15:1317-1326.

14. Ercolini AM, Miller SD: Mechanisms of immunopathology in murine models of central nervous system demyelinating disease. J Immunol 2006, 176:3293-3298.

15. Kanwar JR: Anti-inflammatory immunotherapy for multiple sclerosis/experimental autoimmune encephalomyelitis (EAE) disease. Curr Med Chem 2005, I 2:2947-2962.

16. Peira E, Marzola P, Podio V, Aime S, Sbarbati A, Gasco MR: In vitro and in vivo study of solid lipid nanoparticles loaded with superparamagnetic iron oxide. J Drug Target 2003, I I: 1 9-24.

17. Dupas B, Berreur M, Rohanizadeh R, Bonnemain B, Meflah K, Pradal G: Electron microscopy study of intrahepatic ultrasmall superparamagnetic iron oxide kinetics in the rat. Relation with magnetic resonance imaging. Biol Cell 1999, 91:195-208.

18. Vinogradov SV, Batrakova EV, Kabanov AV: Nanogels for oligonucleotide delivery to the brain. Bioconjug Chem 2004, I5:50-60.

19. Zhang Y, Calon F, Zhu C, Boado RJ, Pardridge WM: Intravenous nonviral gene therapy causes normalization of striatal tyrosine hydroxylase and reversal of motor impairment in experimental parkinsonism. Hum Gene Ther 2003, 14:1-12.

20. Pathak S, Cao E, Davidson MC, Jin S, Silva GA: Quantum dot applications to neuroscience: new tools for probing neurons and glia. J Neurosci 2006, 26:1893-1895.

21. Silva GA: Nanotechnology approaches for drug and small molecule delivery across the blood brain barrier. Surg Neurol 2007, 67: II3-II6.

Publish with Bio Med Central and every scientist can read your work free of charge

"BioMed Central will be the most significant development for disseminating the results of biomedical research in our lifetime. "

Sir Paul Nurse, Cancer Research UK

Your research papers will be:

- available free of charge to the entire biomedical community

- peer reviewed and published immediately upon acceptance

- cited in PubMed and archived on PubMed Central

- yours - you keep the copyright
BioMedcentral 